Stoiber, Edmund (1999): Hochwasser Pfingsten 1999, Regierungserklärung des Bayerischen Ministerpräsidenten vor dem Bayerischen Landtag am 8. Juni 1999

Tversky, Amos / Kahnemann, Daniel (1973): Availability: A Heuristic for Judging Frequency and Probability, in: Cognitive Psychology, Jg. 5, S. 207-232

Ungern-Sternberg, Thomas von (2002): Gebäudeversicherung in Europa. Die Grenzen des Wettbewerbs, Bern

Ungern-Sternberg, Thomas von (200I): Die Vorteile des Staatsmonopols in der Gebäudeversicherung: Erfahrungen aus Deutschland und der Schweiz, in: Perspektiven der Wirtschaftspolitik, Jg. 2, H. I, S. 3I-33

\title{
Die EuroMemorandum-Gruppe: Für einen wirtschaftspolitischen Kurswechsel in Europa \\ The EuroMemorandum Group: For a Change in Economic Policy in Europe
}

Jörg Huffschmid*

\section{Hintergrund}

Dass die Europäische Union (EU) seit Jahren in einer tiefen Krise steckt, liegt zu einem erheblichen Teil an der neoliberalen Wirtschafts- und Sozialpolitik, die sich in den meisten - nicht in allen! - Mitgliedsländern und den Institutionen der EU während der letzten beiden Jahrzehnte weitgehend durchgesetzt hat. Wesentliche Stationen hierbei waren das Binnenmarktprojekt (1985), der Vertrag von Maastricht (1992), der Stabilitäts- und Wachstumspakt (1997) und die Währungsunion (1999). Wäre es nach den Plänen der politischen Klasse gegangen, dann hätte der Neoliberalismus sogar Verfassungsrang für die gesamte EU erhalten. Dies haben die Referenden in Frankreich und den Niederlanden im Mai/Juni 2005 zunächst verhindert. Das Projekt aber ist nicht tot, sondern soll nach den Plänen mächtiger Hardliner im ersten Halbjahr 2007 - wenn Deutschland die Ratspräsidentschaft der EU inne hat - erneut auf die politische Tagesordnung kommen und im zweiten Halbjahr 2008 - dann liegt die Präsidentschaft bei Frankreich - doch noch durchgesetzt werden. Die Vertreter dieser Strategie in Wirtschaft, Politik und Wissenschaft stellen sie als die einzige Möglichkeit dar, Europa aus der mittlerweile lange anhaltenden Periode ökonomischer Schwäche herauszuführen, seine Wettbewerbsfähigkeit in der globalisierten Weltwirtschaft zu stärken und das »europäische Sozialmodell« zu verteidigen.

* Universität Bremen. 
Diese strategische Ausrichtung Europas ist allerdings nicht unwidersprochen geblieben. Der Widerspruch gründet sich erstens auf die wissenschaftliche Kritik an den theoretischen Grundlagen der europäischen Wirtschafts- und Sozialpolitik. Er wird zweitens durch die praktischen Erfahrungen von Wachstumsschwäche, Sozialabbau und zunehmender sozialer Polarisierung in der Bevölkerung in den meisten Ländern der EU gestützt. Diese Erfahrungen haben einerseits dazu geführt, dass die Identifikation vieler Menschen mit Europa stark zurückgegangen ist, was sich z.B. in der sinkenden Beteiligung an den Wahlen zum europäischen Parlament auf zuletzt (2004) 45 Prozent ausdrückt - US-amerikanische Verhältnisse in der EU. Die bisherigen Erfahrungen haben andererseits aber auch den Boden für sozialen und politischen Protest und Gegenbewegungen bereitet, die sich in den einzelnen Ländern in unterschiedlichen Formen und Intensitäten entwickeln. Vielfältige Proteste gegen Privatisierungen, die Demonstrationen gegen die europäische Dienstleistungsrichtli nie, die Streiks gegen Sozialabbau und -dumping haben den neoliberalen Trend zwar noch nicht gebrochen oder gar umgekehrt. Sie haben aber Sand in sein Getriebe geworfen und seine menschenfeindlichen Seiten einer größeren Öffentlichkeit sichtbar gemacht.

Die Gründung EuroMemorandum-Gruppe (EMG) ist im Zusammenhang mit dieser kritischen Gegenbewegung zur neoliberalen Formierung der EU zu sehen. Sie besteht aus professionellen Wirtschafts- und Sozialwissenschaftler|inne|n, die mit den Mitteln der Wissenschaft drei Ziele verfolgen: Sie wollen erstens die theoretische und empirische Kritik vertiefen und verbreitern und zur Widerlegung der Behauptung beitragen, es handele sich beim Neoliberalismus um einen Sachzwang der Geschichte, dessen Durchsetzung sowohl alternativlos als auch zum Besten aller sei. Sie wollen zweitens Konzeptionen und Vorschläge für eine andere Wirtschafts- und Sozialpolitik in der EU entwickeln, die zur Förderung von nachhaltiger Entwicklung und sozialem Zusammenhalt beitragen und so das »europäische Sozialmodell« als Alternative zum angelsächsischen Neoliberalismus stärken. Drittens wollen sie ihre Kritik und Vorschläge in die aktuellen politischen Diskussionen und sozialen Bewegungen einbringen und damit zur Veränderung der wirtschafts- und gesellschaftspolitischen Kräfteverhältnisse in der EU beitragen. Ihre Adressatinnen sind die Wissenschaft, die kritische Öffentlichkeit und die sozialen Bewegungen gegen den Neoliberalismus.

\section{Entstehung, Struktur und Verfahren der EMG}

Unter dem Eindruck zunehmender Formierung der EU nach den Beschlüssen des Gipfels von Maastricht (I99I) und vor der geplanten Europäischen Währungsunion trafen sich vom I3. bis I5. Oktober I995 ca. zwei Dutzend Wirtschaftswissenschaftler|innen aus einem halben Dutzend Mitgliedsländern der EU in einem Raum des Europäischen Parlamentes (EP) in Straßburg, der von einem Mitglied der Grünen Fraktion im EP zur Verfügung gestellt worden war. Die Initiative zu diesem »Workshop for Alternative Economic Policy in Europe« ging von deutschen Kollegen aus, sie war auch inspiriert durch die positiven Erfahrungen mit der "Arbeitsgruppe Alternative Wirtschaftspolitik" (Memorandum-Gruppe) in Deutschland, die seit 1975 jedes Jahr in einem größeren Gutachten die vorherrschende Wirtschafts- und Sozialpolitik kritisiert und Vorschläge für eine andere Politik vorlegt. Die 
Zusammensetzung des Treffens in Straßburg war durch persönliche Kontakte und Bekanntschaften bestimmt. In den Diskussionen zeigte sich zweierlei: Erstens waren bei allen Unterschieden in den einzelnen Ländern bestimmte Probleme sehr ähnlich, insbesondere die mit der radikalen Marktöffnung verbundene Tendenz zur Deregulierung und die durch den Vertrag von Maastricht in Gang gesetzte Knebelung der Makropolitik, v.a. der Geld- und der Fiskalpolitik. Zweitens gab es in den einzelnen Ländern zwar Kritik und Widerspruch hiergegen, es war aber trotz der zunehmend gemeinsamen Probleme nicht zu einer gemeinsamen europäischen Kritik gekommen. Um eine solche Kritik und Alternativvorschläge auf europäischer Ebene voranzubringen, beschlossen die Teilnehmer|innen des Straßburger Workshops, sich regelmäßig zu treffen, gemeinsame Positionen zu entwickeln und diese in die Öffentlichkeit zu tragen. Die erste Frucht dieser Bemühungen war ein Memorandum "Vollbeschäftigung, sozialer Zusammenhalt und Gerechtigkeit - Für eine alternative Wirtschaftspolitik in Europa«. Es wurde im Mai 1997, im unmittelbaren Vorfeld des Gipfels von Amsterdam (Juni 1997), in fünf europäischen Städten - Amsterdam, Athen, Barcelona, Paris und Bonn - veröffentlicht. Eine Besonderheit dieses Memorandums bestand darin, dass es die Unterschriften von mehr als 250 europäischen Wirtschaftswissenschaftler|inne|n aus zehn der I5 Mitgliedsländer der EU Ländern trug. Dies zeigte, dass es eine europaweite Grundlage für Kritik an der vorherrschenden Wirtschaftspolitik in Europa und für Alternativvorschläge hierzu gab.

Die EMG ist mittlerweile zu einem breiteren Netzwerk geworden. Sie veranstaltet jedes Jahr einen größeren Workshop (den zwölften Ende September 2006), an dem 50 bis 70 Personen aus mehr als einem Dutzend Mitgliedsländern der EU teilnehmen. Dort werden u.a. auch die Inhalte des jährlichen Memorandums diskutiert, das in der Regel kurz vor dem Dezember-Gipfel der EU erscheint und von 250 bis 350 Ökonom|inn|en durch ihre Unterschrift unterstützt wird. Diese Memoranden stehen auf der Homepage der Gruppe - www.memo-europe.uni-bremen.de - in acht Sprachen zur Verfügung und werden von den nationalen Unterstützer|inne|n je nach deren Kräften und Interessen verbreitet. In Deutschland erscheint das jährliche Memorandum seit 2003 auch als Buch im VSA Verlag zusammen mit ergänzenden Artikeln aus dem Umkreis der Gruppe.

Es gibt keine strenge Struktur der EMG, keine formale Mitgliedschaft, keine Satzung, keine Kassierer - und kein öffentliches Geld. In den ersten zehn Jahren wurde sie in der Universität Bremen koordiniert, seit 2005 gibt es ein kleines »steering committee«, das auf den Jahreskonferenzen gewählt wird. Die enge Zusammenarbeit mit verschiedenen wissenschaftlichen Netzwerken, die von der EU im Rahmen ihrer Forschungspolitik gefördert werden, hat gelegentlich die infrastrukturellen Bedingungen für die großen Arbeitskonferenzen sowie das Treffen einzelner Arbeitsgruppen erleichtert, ohne die Unabhängigkeit der Gruppe im geringsten zu beeinträchtigen.

\section{Schwerpunkte}

Die Kritik der EMG an der neoliberalen Orientierung in der EU und die Ausarbeitung von Alternativvorschlägen haben sich bisher auf folgende Gebiete konzentriert: 


\section{a. Makroökonomischer Fundamentalismus in der Geld-und Fiskalpolitik}

Die EMG kritisiert, dass sich die von der Europäischen Zentralbank (EZB) für die Länder der Währungsunion betriebene Geldpolitik ausschließlich am Ziel der - zudem noch äußerst restriktiv definierten - Preisstabilität (»unter aber nahe an zwei Prozent«) ausrichtet und jede Verantwortung für Wachstum und Beschäftigung ablehnt. Die völlige Unabhängigkeit der EZB entzieht sie überdies jeder Einflussnahme durch politische Diskussionen und demokratische Parlaments- oder Regierungsentscheidungen. Beides ist nicht akzeptabel. Demgegenüber vertritt die EMG die Position, dass die Geldpolitik in der EU auch beschäftigungs- und andere wirtschaftspolitische Ziele (z.B. stärker als bisher den Wechselkurs des Euro) berücksichtigen und daher in enger Abstimmung mit der übrigen Wirtschaftspolitik in der EU erfolgen soll. Dabei sind jeweils Kompromisse zwischen den verschiedenen Zielen erforderlich, die einer demokratischen Legitimierung bedürfen. Die EMG kritisiert an den Vorgaben der EU für die Fiskalpolitik deren vorrangige Orientierung an dem Kriterium des Ausgleichs der öffentlichen Haushalte. Diese Orientierung ist ökonomisch kontraproduktiv, sie wirkt makroökonomisch prozyklisch und sozial polarisierend. Stattdessen sollte der öffentliche Haushalt als Instrument zur makroökonomischen Stabilisierung, zum sozialen Ausgleich und zur Bereitstellung öffentlicher Güter auf nationaler wie europäischer Ebene eingesetzt werden. Dazu bedarf es einer sehr viel besseren und verbindlicheren Koordination zwischen den Mitgliedsländern. Es bedarf aber auch eines größeren finanziellen Spielraumes der EU-Ebene durch die Ausweitung des EU-Haushaltes, der sich gegenwärtig nur auf ungefähr ein Prozent des EU-Bruttoinlandsprodukts beläuft und in dieser Höhe für die nächsten Jahre eingefroren werden soll. Damit lässt sich keine relevante europäische Wirtschaftspolitik machen.

\section{b. Liberalisierungs-, Deregulierungs- und Privatisierungsradikalismus}

Hier markiert das Binnenmarktprojekt von 1985 einen qualitativen Einschnitt: Die ursprüngliche Konzeption, zugleich mit der Herstellung eines gemeinsamen Binnenmarktes die unterschiedlichen Rechtsvorschriften in der EU weitgehend zu harmonisieren, wurde durch das Prinzip der gegenseitigen Anerkennung der jeweiligen Rechtsvorschriften jedes Mitgliedslandes, oder das Prinzip der "positiven « durch das der »negativen« Integration, ersetzt. Dies bedeutet eine weitgehende Veränderung des Charakters ökonomischer Konkurrenz: An die Stelle der Konkurrenz von Unternehmen um das Geld der Kund|inn|en mit ökonomischen Parametern wie Produktivität, Markterschließung etc., also den Schumpeterschen Innovationen, tritt zunehmend die Konkurrenz der Staaten um die Unternehmen mit Parametern wie Steuern, Sozialabgaben, Arbeits- und Umweltschutz etc. An die Stelle der Konkurrenz innerhalb eines gemeinsamen Rahmens akzeptierter Regeln tritt die Konkurrenz der Regeln. Der Leistungswettbewerb wird durch den Standortwettbewerb ergänzt und zunehmend ersetzt. Gleichzeitig werden die Wettbewerbsregeln immer mehr auch auf öffentliche Dienstleistungen angewandt. Dies hat die massive Privatisierungswelle gefördert, die wir seit den I99oer Jahren beobachten. Die Zunahme von sozialer Ungleichheit und Polari- 
sierung sind die Folge. Als Alternative hierzu fordert die EMG zum einen die politische Herstellung eines Rahmens gemeinsamer Regeln für den europäischen Binnenmarkt, als Zwischenschritte die Entwicklung gemeinsamer Mindeststandards und die Anwendung des Gastlandprinzips bis zur Vereinheitlichung der Regeln. Zum anderen fordert sie, den öffentlichen Sektor zu stärken und zu demokratisieren - nicht als eng begrenzte und immer attackierte Ausnahme vom ansonsten universell geltenden Prinzip des privaten Wettbewerbs, sondern als eigenständigen Bereich mit eigenen Regeln, über dessen Umfang und Inhalt politisch entschieden wird.

c. Disziplinierung in der Arbeitsmarkt-und Sozialpolitik

Obgleich die EU für Sozial- und Arbeitsmarktpolitik nicht zuständig ist, hat sie in beiden Bereichen politische Initiativen gestartet, die eine große Wirkung haben. Sie hat hierzu ein im EU-Vertrag nicht vorgesehenes Verfahren der "offenen Methode der Koordinierung « entwickelt, über das Druck auf die Mitgliedsländer ausgeübt wird, ihre Arbeitsmärkte stärker zu deregulieren und »beschäftigungsfeindliche« sozialpolitische Regelungen abzuschaffen: durch Kürzungen der Unterstützung für Arbeitslose und Abschaffung oder Verschlechterung der Möglichkeiten des Vorruhestandes. Starken Druck übt die EU auch in Richtung (Teil-)Privatisierung der überwiegend umlagefinanzierten Rentensysteme in der EU aus. Durch beides wird die soziale Situation der Betroffenen verschlechtert. In der Folge dieser Politik nimmt die Armut in der EU mittlerweile wieder zu. Demgegenüber fordert die EMG eine Arbeitsmarkt- und Beschäftigungspolitik, die sich auf die Schaffung neuer und attraktiver Arbeitsplätze konzentriert, sowie die Stärkung der umlagefinanzierten Alterssicherungssysteme und eine sehr viel energischere Bekämpfung der Armut in der EU - auch auf zentraler Ebene.

Neben diesen Zentralbereichen, mit denen sich die EMG kontinuierlich beschäftigt, gibt es einige andere wesentliche Themen, mit denen sich die Gruppe nur gelegentlich befasst hat und bei denen weitere intensive Beschäftigung erforderlich und in Zukunft immer dringlicher sein wird. Dazu gehören vor allem Fragen der ökologischen Nachhaltigkeit. Erforderlich ist sowohl eine Kritik an den unzureichenden und zum Teil kontraproduktiven Konzepten der EU als auch eine Konzeption, die gleichermaßen den ökologischen Umbau, Vollbeschäftigung und soziale Sicherheit umfasst. Auf diesem Gebiet sind verstärkte Kooperationen mit Gruppen und Netzwerken erforderlich, die hierzu schon länger arbeiten. Weitgehend unbearbeitet geblieben sind bisher auch Fragen der internationalen Wirtschaftsbeziehungen der EU mit den Zentralfeldern Handelspolitik und Entwicklungspolitik. Hier bleibt viel zu tun.

\section{Theoretische Grundlagen: linker Eklektizismus}

Eine ausgearbeitete einheitliche theoretische Grundposition hat die EMG nicht. Das muss kein Nachteil sein. Denn einerseits sind die vorhandenen einheitlichen Theorien "aus einem Guss" offensichtlich nicht in der Lage, die gegenwärtige Wirklichkeit überzeugend zu erklären und daraus sinnvolle wirtschaftspolitische Vorstellungen abzuleiten. Zum anderen ist 
der theoretisch mehr eklektische Charakter der EMG von Vorteil, weil er eine breite wissenschaftliche und wirtschaftspolitische Zusammenarbeit ermöglicht. Die Mitglieder kommen aus unterschiedlichen wirtschaftstheoretischen Zusammenhängen und wirtschaftspolitischen Orientierungen. Was sie eint, ist die Kritik an der vorherrschenden neoliberalen Ausrichtung der Wirtschafts- und Sozialpolitik in der EU und der Wille, Vorschläge für eine andere Ausrichtung zu entwickeln und wissenschaftlich zu begründen. Beides ist von verschiedenen theoretischen und wirtschaftspolitischen Grundpositionen aus möglich.

Es gibt verschiedene Haupttraditionen, die in den Veröffentlichungen der EMG erkennbar werden. Dazu gehören vor allem:

- Die keynesianische Makroökonomie mit ihrer Betonung der immanenten Instabilität kapitalistischer Systeme und der Notwendigkeit politischer Stabilisierung und die sich hierauf beziehenden Theorievarianten in der Tradition Hyman Minskis oder Michal Kaleckis;

- die Marxsche Theorie kapitalistischer Akkumulation, Entwicklungsdynamik, Krisen und die darauf aufbauenden Theorien der Veränderungen auch des Arbeitsprozesses;

- die Theorien des Sozialstaates, etwa in der Tradition von Lord William Beveridge oder Gunnar Myrdal;

- die Theorien über die sozialen und ökologischen Kosten der Marktwirtschaft in der Tradition von William Kapp, die das Problem der Zerstörung durch die Beschränkung auf das mikroökonomische Profitstreben betonen und erstmals das Konzept einer nachhaltigen Entwicklung vertreten;

- die Theorien institutioneller Einbettung der Wirtschaft in der Tradition von Karl Polyani.

Die Heterogenität der EMG beruht nicht nur auf der Unterschiedlichkeit der wirtschaftstheoretischen und wirtschaftspolitischen Denkschulen und -traditionen. Sie reflektiert auch die Unterschiede zwischen den einzelnen Ländern und Regionen der EU. Diese Differenzen bestehen nicht nur in der wirtschaftlichen und sozialen Entwicklung und den wirtschaftstheoretischen Hauptorientierungen in diesen Ländern - diese werden durch den Neoliberalismus gerade in gewissem Maße eingeebnet. Sie zeigen sich auch bei der Kritik und der Konzeption von Alternativen, die z.B. von sehr staatsskeptisch bis sehr staatszentriert reichen.

In theoretisch letzter Instanz gibt es zwischen den in der EMG vertretenen Positionen nicht nur Unterschiede, sondern auch Unvereinbarkeiten. Nur: die EMG arbeitet nicht auf der Ebene der letzten Instanz, sondern in einem Bereich mittlerer theoretischer und politischer Reichweite, in dem theoretisch »letztinstanzlich unvereinbare " Positionen gemeinsamen Boden für Kritik und Alternativvorschläge finden. Die theoretischen Widersprüche führen zu Kontroversen im Rahmen der EMG - etwa über die Rolle des Wachstums, der Atomenergie, der Unternehmenskonzentration. Sie werden auf den Jahreskonferenzen und den Arbeitsgruppensitzungen ausgetragen. In den Memoranden finden sich dann Kompromisse auf Basis eines gemeinsamen Nenners. Das macht die Formulierungen manchmal ein bisschen ungenau und schwammig, was der Preis einer breiten Basis ist. 
Die EMG hat sich in ihrem Memorandum 2004 mit dem Entwurf für eine europäische Verfassung auseinandergesetzt, diesen scharf kritisiert und abgelehnt. Der Grund für diese Ablehnung ist, dass mit der Verfassung die neoliberale Grundausrichtung, die sich in der Wirtschafts- und Sozialpolitik der EU in den letzten beiden Jahrzehnten zunehmend durchgesetzt hat, festgeschrieben werden und Verfassungscharakter erhalten soll. Dies ist unwissenschaftlich und undemokratisch. Es läuft darauf hinaus, eine theoretische Position, die sehr umstritten ist und zunehmend kritisiert wird, praktisch gegen diese Kritik zu immunisieren, der Kritik und damit auch der Veränderung zu entziehen. Die Gegenposition der EMG ist nicht die Forderung nach einer keynesianischen Orientierung der Wirtschaftspolitik in der Verfassung. Vielmehr sollte die Verfassung grundsätzlich für verschiedene wirtschaftspolitische Optionen offen sein. Sie sollte sich auf die Festlegung von Zielen (Solidarität, Gerechtigkeit, soziale Sicherheit, Vollbeschäftigung, Nachhaltigkeit) und auf die Definitionen der demokratischen Institutionen und Regeln beschränken, mit denen diese Ziele verfolgt werden sollen. Dies erlaubt es, wirtschaftspolitische Positionen öffentlich zu diskutieren und zu verändern, wenn neue Erkenntnisse sich durchsetzen und/oder neue politische Mehrheiten zustande kommen.

\section{Erfolg und Perspektiven}

Positiv ist zu verbuchen, dass die Gruppe sich bei den kritischen Wirtschaftswissenschaftler|inne|n, die sich mit der EU beschäftigen, gut etabliert hat. Dies belegt u.a. die Zahl von zuletzt immer mehr als 300 Unterstützer|inne|n für die jährlichen Memoranden. Die kontinuierliche Arbeit hat auch dazu beigetragen, die Kritik auszuweiten und zu vertiefen und eine relativ solide Systematik der Forderungen nach einer anderen Wirtschaftspolitik zu entwickeln. Die Wahrnehmung in der Öffentlichkeit ist sehr unterschiedlich. In den europäischen Institutionen Rat und Kommission wird die Gruppe schlicht nicht zur Kenntnis genommen, im EP nur - aber immerhin - von der kleinen linken Fraktion unterstützt. In einzelnen Ländern - Griechenland, Deutschland, Frankreich u.a. - gibt es in der Regel ein kurzes Presseecho auf die Veröffentlichung der Memoranden, in anderen Ländern, z.B. Großbritannien, Spanien, ist dies nicht der Fall. In den Gewerkschaften wird das Memorandum auf europäischer Ebene wahrgenommen und diskutiert, in einzelnen Ländern darüber hinaus auch für Seminare und ähnliche Veranstaltungen herangezogen. Auch in anderen sozialen Bewegungen ist die Gruppe präsent, z.B. auf den europäischen Sozialforen in Florenz, Paris und Athen. Im April 2006 hat sie sich intensiv an einem von attac Österreich, den österreichischen Gewerkschaften und anderen Gruppen organisierten öffentlichkeitswirksamen Gegengipfel zum offiziellen Treffen des Europäischen Rates für Wirtschaft und Finanzen (Ecofin) beteiligt. Ähnliche Veranstaltungen sollten regelmäßig stattfinden.

Die Perspektiven für eine größere Verbreitung der Positionen der EMG hängen zum Teil natürlich von der Fähigkeit dieser Gruppe ab, ihre Kritik und Alternativvorschläge weiter zu entwickeln und in der Öffentlichkeit überzeugend vorzutragen. Längerfristig hängen sie allerdings in erster Linie von der wirtschafts- und sozialpolitischen Großwetterlage in der EU ab. Diese wird einerseits durch den nicht nachlassenden Druck des Neoliberalis- 
mus und der hinter ihm stehenden Macht- und Interessengruppen und andererseits durch den Gegendruck bestimmt, der von einer zunehmend kritischen Öffentlichkeit und stärker werdenden sozialen Bewegungen ausgeht.

\section{Aktuelle Publikationen}

Etxezarreta, Miren / Grahl, John / Huffschmid, Jörg / Mazier, Jacques (2003): Bessere Institutionen, Regeln und Instrumente für Vollbeschäftigung und sozialen Wohlstand in Europa, EuroMemo 2002, Hamburg

Etxezarreta, Miren / Grahl, John / Huffschmid, Jörg/ Mazier, Jacques (2004): Vollbeschäftigung, Wohlfahrt und ein starker öffentlicher Sektor. Demokratische Herausforderungen in einer erweiterten Union, EuroMemo 2003, Hamburg

Etxezarreta, Miren / Grahl, John / Huffschmid, Jörg/ Mazier, Jacques (2005): Jenseits von Lissabon. Wirtschafts- und sozialpolitische Leitlinien und Eckpunkte einer Verfassung für das europäische Gesellschaftsmodell, EuroMemo 2004, Hamburg

Etxezarreta, Miren / Grahl, John / Huffschmid, Jörg/Mazier, Jacques (2006): Demokratische

Politik gegen die Herrschaft der Märkte. Vorschläge für eine integrierte Entwicklungsstrategie in Europa, EuroMemo 2005, Hamburg

Huffschmid, Jörg (Hg.) (2005): Economic Policy for a Social Europe - A Critique of Neo-liberalism and Proposals for Alternatives, Houndmills: Palgrave Macmillan

\section{Information und Kontakt}

www.memo-europe.uni-bremen.de

Auf der Website verfügbar sind die meisten Memoranden in voller Länge, Kurzversionen in bis zu acht Sprachen sowie ergänzende Beiträge aus dem Umkreis der EuroMemorandumGruppe. 